\title{
Two distinct dielectric relaxation mechanisms in the low-frequency range in $\mathrm{Bi}_{5} \mathrm{TiNbWO}_{15}$ ceramics
}

\author{
Z. G. Yi, ${ }^{a}$ Y. X. Li, ${ }^{\text {b) }}$ Y. Wang, ${ }^{\text {a) }}$ and Q. R. Yin \\ State Key Laboratory of High Performance Ceramics and Superfine Microstructures, Shanghai \\ Institute of Ceramics, Chinese Academy of Sciences, 1295 Dingxi Road, Shanghai 200050, \\ People's Republic of China
}

(Received 7 October 2005; accepted 21 February 2006; published online 21 April 2006)

\begin{abstract}
The ac data in terms of impedance and dielectric loss $(\tan \delta)$ were exploited simultaneously to probe the dielectric relaxation mechanisms in $\mathrm{Bi}_{5} \mathrm{TiNbWO}_{15}$ ceramics. It was found that two distinct relaxation mechanisms exist in the low-frequency range $(10 \mathrm{~Hz}-5 \mathrm{MHz})$. One is attributed to the grain boundary relaxation and the other is associated with oxygen ion diffusion. Furthermore, the temperature dependence of the oxygen vacancy relaxation strength is analogous to the Curie-Weiss law and follows the traditional point defect relaxation theory. These results could be helpful to understand the phenomena related to ferroelectric fatigue, oxygen ion conductivity, etc. (C) 2006 American Institute of Physics. [DOI: 10.1063/1.2191953]
\end{abstract}

The intergrowth bismuth layer-structured ferroelectrics (IBLSFs) were first reported in 1970s. ${ }^{1}$ However, it is only recently that the potential of this kind of materials for device applications has begun to be realized. $^{2-4}$ Moreover, the unique natural superlattice structures of this kind of compounds, implying other superior properties, renews interest in the fundamental solid-state chemistry and physics of the Aurivillius phases. ${ }^{5,6}$ For example, the compound $\mathrm{Bi}_{5} \mathrm{TiNbWO}_{15}$, which is built up by a regular intergrowth of one-half the unit cell of $\mathrm{Bi}_{2} \mathrm{WO}_{6}$ and one-half the unit cell of $\mathrm{Bi}_{3} \mathrm{TiNbO}_{9}$ along the $c$ axis, is not only a ferroelectric, but also a superior oxygen ion conductor, ${ }^{7}$ and has potential applications in nonvolatile ferroelectric random memory (FeRAM), solid oxide full cells (SOFC), chemical sensors, etc.

It is well known that solid defects have great influence on the performances of materials. For example, oxygen vacancies, the dominant point defects in Aurivillius phase compounds, play an important role in ferroelectric fatigue or ionic conductivity. ${ }^{8}$ It is of great importance to gain a fundamental understanding of the defects in solids. In this Letter, the ac complex impedance technique was employed to probe the dielectric relaxation mechanisms in $\mathrm{Bi}_{5} \mathrm{TiNbWO}_{15}$ ceramics. The results are helpful to shed light on the defects dynamics within the compound.

The wafer specimens of $\mathrm{Bi}_{5} \mathrm{TiNbWO}_{15}$ with a diameter of $13 \mathrm{~mm}$ and a thickness of $1 \mathrm{~mm}$ were prepared by conventional solid-state reaction method. Appropriate amounts of high purity raw materials (in which excess $\mathrm{Bi}_{2} \mathrm{O}_{3}$ of $2 \mathrm{~mol} \%$ out of the stoichiometry was added to compensate for the volatilization of bismuth oxide during sintering at high temperatures) were first mixed and presintered at $1173 \mathrm{~K}$ for $7 \mathrm{~h}$, then pressed into pellets and sintered at $1313 \mathrm{~K}$ in air for $2 \mathrm{~h}$. The $\mathrm{x}$-ray diffraction (XRD) patterns of sintered specimens showed monophasic compound, and can fully indexed on the lattice cell reported in Ref. 6. The impedance spectra were performed on Pt-pasted samples, in

\footnotetext{
a) Also at Graduate School of the Chinese Academy of Sciences, Beijing 100039, People's Republic of China.

b) Electronic mail: yxli@mail.sic.ac.cn
}

the frequency range of $10 \mathrm{~Hz}-5 \mathrm{MHz}$, by an HP4192A impedance analyzer in air.

Figure 1 shows the frequency dependence of the imaginary part of impedance, at temperatures between 393 and $673 \mathrm{~K}$ at intervals of $20 \mathrm{~K}$. When temperature equals $393 \mathrm{~K}$, one peak of $Z^{\prime \prime}(\omega)$ (denoted as $P_{\mathrm{Z} 1}$ ) appears unconspicuously, which shifts to higher-frequency range with increasing temperature. Simultaneously, when temperature increases to $473 \mathrm{~K}$, another peak of $\mathrm{Z}^{\prime \prime}(\omega)$ (denoted as $P_{\mathrm{Z} 2}$ ) emerges at lower-frequency decades, which also shifts to higherfrequency range with increasing temperature. However, when temperature is above $573 \mathrm{~K}$, the $P_{\mathrm{Z} 1}$ peak becomes very weak and nearly shifts out of the frequency range. These phenomena indicate that there are two thermally activated relaxation processes within the measured frequency range of $10 \mathrm{~Hz}-5 \mathrm{MHz}$.

For a thermally activated relaxation process, the temperature dependence of the relaxation time follows the Arrhenius law: $\tau=\tau_{0} \exp \left(E / K_{B} T\right)$, where $\tau_{0}$ is the prefactor, $E$ denotes the activation energy, $K_{B}$ is the Boltzman constant, and $T$ is the absolute temperature. The Arrhenius plots for the two dielectric loss peaks are shown in Fig. 2. From this figure, the relaxation parameters $E_{1}=0.82 \mathrm{eV}$ and

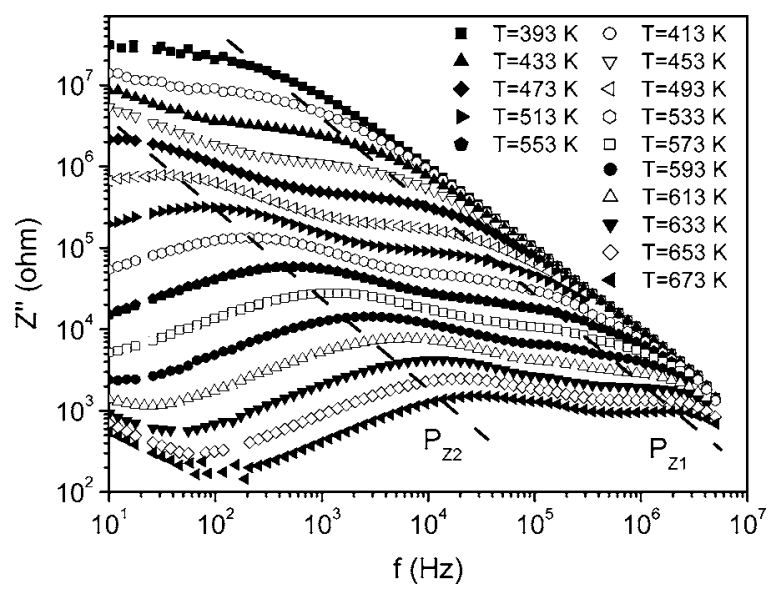

FIG. 1. Imaginary part of the impedance as a function of frequency at various temperatures for the $\mathrm{Bi}_{5} \mathrm{TiNbWO}_{15}$ ceramic. 


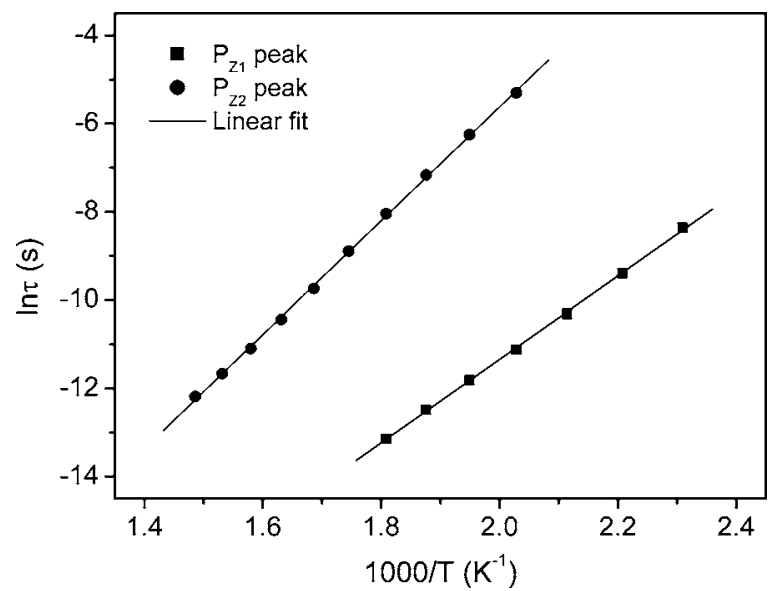

FIG. 2. Arrhenius plot of the two relaxation peaks $\left(P_{\mathrm{Z} 1}\right.$ and $\left.P_{\mathrm{Z} 2}\right)$ from the frequency dependence of the imaginary part of the impedance. The solid lines are linear least-square fittings.

$\tau_{01}=7.3 \times 10^{-14} \mathrm{~s}$ for the $P_{\mathrm{Z1}}$ peak, and $E_{2}=1.11 \mathrm{eV}$ and $\tau_{02}=2.2 \times 10^{-14} \mathrm{~s}$ for the $P_{\mathrm{Z} 2}$ peak are obtained from these fitting lines. The discrepancies for the relaxation parameters with our previous results ${ }^{7}$ indicate that the relaxation processes are influenced by sample preparation, especially the one at lower frequency.

Figure 3 presents the impedance spectra of the $\mathrm{Bi}_{5} \mathrm{TiNbWO}_{15}$ specimen measured at $553 \mathrm{~K}$ in air. From high frequency to low frequency, there are two semicircles attributed to bulk (grain) and grain boundary relaxation, respectively. However, from the inset in Fig. 3, impedance spectra of the $\mathrm{Bi}_{5} \mathrm{TiNbWO}_{15}$ specimen measured at a lower temperature of $413 \mathrm{~K}$, only part of one semicircle attributed to bulk relaxation, are observed in the same frequency range. As a result, we can attribute the $P_{\mathrm{Z1}}$ peak to the relaxation of the bulk and the $P_{\mathrm{Z} 2}$ peak to the grain boundary effects.

Figure 3 also shows the fitting results of the impedance spectra measured at $553 \mathrm{~K}$. The two fitted curves are obtained experientially by fitting the experimental data with two semicircles. The resistances and capacitances of grains and grain boundaries can be deduced from the fitting results accordingly. Let $\left(R_{g}, R_{\mathrm{gb}}\right)$ and $\left(C_{g}, C_{\mathrm{gb}}\right)$ be the resistances and capacitances of grains and grain boundaries, respectively. The corresponding values of these elements are $C_{g}$ $=2.76 \times 10^{-10} \mathrm{~F}$ and $R_{g}=0.043 \mathrm{M} \Omega$ and $C_{\mathrm{gb}}=1.13 \times 10^{-8} \mathrm{~F}$

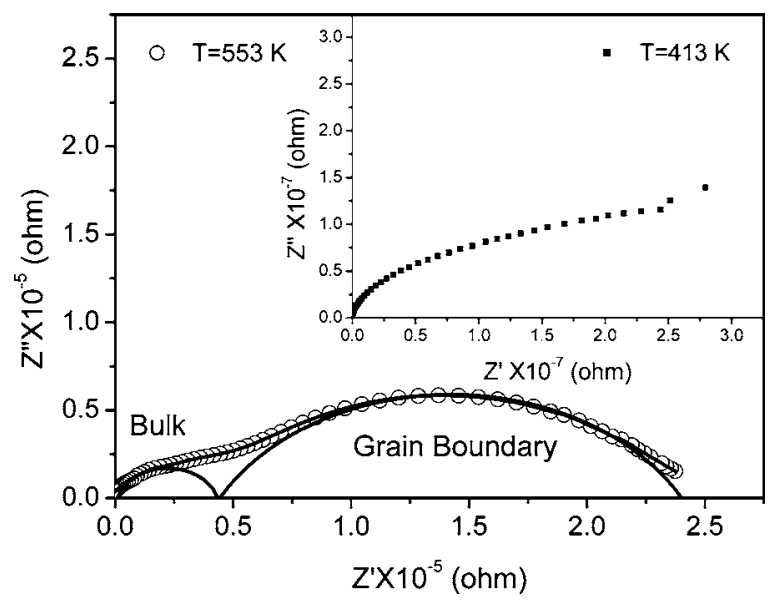

FIG. 3. Complex impedance plot for the $\mathrm{Bi}_{5} \mathrm{TiNbWO}_{15}$ ceramic at temperature of $553 \mathrm{~K}$. The inset is the same plot at a low temperature $413 \mathrm{~K}$.

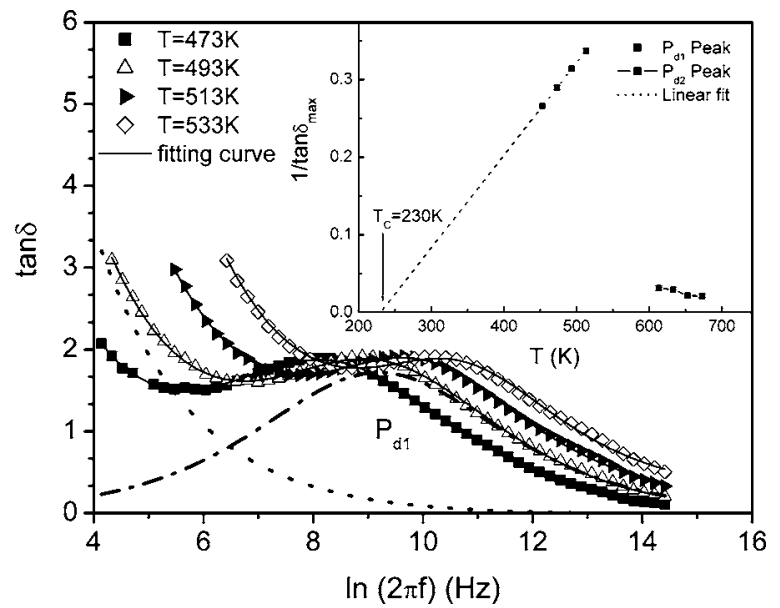

FIG. 4. Frequency dependence of the dielectric loss for the $\mathrm{Bi}_{5} \mathrm{TiNbWO}_{15}$ ceramic measured at different temperatures. The symbols are experimental data points and solid lines are the total fitting curves. The dotted line and dashed-dotted line are fitted background and $P_{\mathrm{d} 1}$ peak, respectively. The inset is the reciprocal of the peak height as function of temperature for the $P_{\mathrm{d} 1}$ peak.

and $R_{\mathrm{gb}}=0.197 \mathrm{M} \Omega$. The $C_{g}$ value is a characteristic one for bulk ferroelectric and the $C_{\mathrm{gb}}$ value reveals the sample is well sintered, with narrow intergranular regions. ${ }^{9}$

To further probe the relaxation mechanism within grain interior, we calculated the dielectric loss $\tan \delta$ from the impedance spectra and showed the corresponding relaxation process of bulk material in Fig. 4. In all of the frequency spectra, a wide and prominent relaxation peak (labeled $P_{\mathrm{d} 1}$ peak) superimposed on a large frequency dependent background is observed and shifts obviously to higher frequency with increasing temperature.

According to the modified Debye equation, ${ }^{10}$ the dielectric loss peak can be deduced as follows:

$$
\tan \delta=\frac{2 \Delta \sin (\beta \pi / 2)}{\cosh (\beta z)+\cos (\beta \pi / 2)},
$$

where $\Delta$ is the relaxation strength, $z=\ln (\omega \tau), \beta=1-\alpha$, and $\alpha$ $(0 \leqslant \alpha<1)$ is the width parameter leading to a symmetric broadening of the Debye relaxation. ( $\alpha=0$ correspo nds to the standard Debye relaxation.) The fitting result of frequency spectra measured at $493 \mathrm{~K}$ is shown also in Fig. 4. It can be seen that a loss peak $\left(P_{\mathrm{d} 1}\right)$ and a frequency dependent background could fit the dielectric loss curve very well, where the width parameter $\beta=0.61$, or $\alpha=0.39$ (The nonzero $\alpha$ means a correlation among the relaxation species. $\left.{ }^{11}\right)$ The background loss comes mainly from the static conduction of the grain, the curve shifts toward higher frequency with increasing temperature, which is a thermally activated process. $^{12}$

Based on above fitting results, we can analyze the variation of the peak height (or relaxation strength) with temperature in detail. The inset in Fig. 4 shows the reciprocal of the peak height of $P_{\mathrm{d} 1}$ peak as a function of temperature. From it a linear relationship is obtained, which reveals that the correlation among the relaxation units under applied electric field is analogous to the interactions among point defects under applied stress field. According to the point defect relaxation theory under a stress field, ${ }^{13}$ the insertion of a point defect into a grain produces local elastic distortions, thus introduce a local stress field. There are interactions between o AIP license or copyright; see http://apl.aip.org/apl/copyright.jsp 


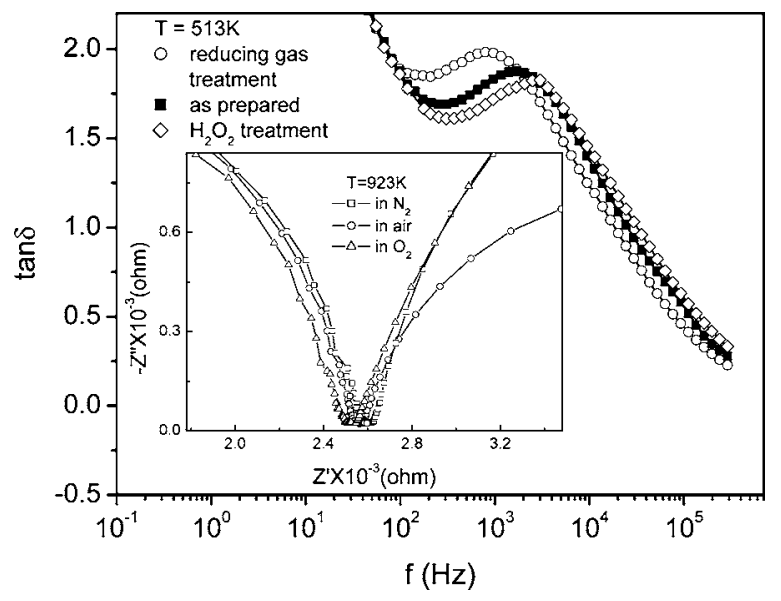

FIG. 5. Dielectric loss as a function of frequency for $\mathrm{Bi}_{5} \mathrm{TiNbWO}_{15}$ ceramics treated under different gas atmospheres at $513 \mathrm{~K}$. The inset is the complex impedance plot under various gas atmospheres measured at $923 \mathrm{~K}$.

the local stress fields and the external stress field applied to the grain, besides, there are interactions among these local stress fields. When the concentration of point defects reach to some extent, the interactions among the defects become unignorable and thus a relation that is analogous to the CurieWeiss law for the temperature dependence of the relaxation strength $\Delta$ is deduced: $\Delta \propto\left(T-T_{c}\right)^{-1}$, where $T_{c}$ is the critical temperature for a "self-induced" ordering, i.e., the temperature at which defects ordering would occur spontaneously, without the presence of an applied stress, if atom movements could take place. In our case, the relaxation strength of the $P_{\mathrm{d} 1}$ peak depends upon temperature obeying the Curie-Weiss law and the critical temperature $T_{c}=230 \mathrm{~K}$ is obtained as labeled by an arrow in the inset of Fig. 4. In view of the similarity between the dielectric relaxation and the mechanical relaxation, the relaxation species corresponding to the $P_{\mathrm{d} 1}$ peak may be of point defects. Furthermore, the $\mathrm{Bi}_{5} \mathrm{TiNbWO}_{15}$ compound is a super oxygen ion conductor at high temperature, the relaxation species might be of oxygen vacancies.

In order to test further whether the $P_{\mathrm{d} 1}$ peak is of oxygen vacancy relaxation, the $\mathrm{Bi}_{5} \mathrm{TiNbWO}_{15}$ sample was cut into three same sheets. One was dipped in $\mathrm{H}_{2} \mathrm{O}_{2}$ solution for $10 \mathrm{~h}$ to fill oxygen into the sample and then baked at $353 \mathrm{~K}$ for $2 \mathrm{~h}$ to remove water in the specimen. Another sheet was annealed under reducing atmosphere $\left(97 \% \mathrm{~N}_{2}\right.$ and $3 \% \mathrm{H}_{2}$ mixed gas) at a temperature of $673 \mathrm{~K}$ for $10 \mathrm{~h}$ to reduce the oxygen content in the specimen. Figure 5 shows the $P_{\mathrm{d} 1}$ peak variation with frequency at $513 \mathrm{~K}$ for the three specimens processed under different oxygen ambients. Compared with the as-prepared one, the dielectric loss peak height of the sample processed in the oxygen-sufficient ambient decreased and the one in the oxygen-deficient ambient increased evidently.

It is well known that the process under the oxygensufficient ambient decreases the density of oxygen vacancies and reversely the process under the oxygen-deficient ambient increases the density of oxygen vacancies in the sample. However, the treatment under various gas atmospheres also changes the concentration of hole or electron. To establish the contribution of electron or hole to the relaxation strength the impedance spectra experiments under different gas atmospheres at $923 \mathrm{~K}$ were performed. From the inset in Fig. 5 it can be seen that the intercept of the impedance spectra and the abscissa shifts only slightly to lower side of resistance with the gas atmospheres change from nitrogen to air and to oxygen. These phenomena indicate that the ceramic is an ionic conductor of $p$-type nature, and the electron or hole contribution is negligible. Therefore, the $P_{\mathrm{d} 1}$ peak was considered to be associated with oxygen vacancy relaxation.

In summary, two distinct dielectric relaxation mechanisms in $\mathrm{Bi}_{5} \mathrm{TiNbWO}_{15}$ ceramic were elucidated from ac impedance and dielectric loss spectra. The obtained results are valuable to the further studies of ferroelectric fatigue and oxygen ion conductivity.

This work was supported by the Ministry of Science and Technology of China through 973-Project (No. 2002CB613307) and the National Natural Science Foundation of China (No. 50572113).

${ }^{1}$ T. Kikuchi, J. Less-Common Met. 48, 319 (1976).

${ }^{2}$ Y. Noguchi, M. Miyayama, and T. Kudo, Appl. Phys. Lett. 77, 3639 (2000).

${ }^{3}$ Y. Noguchi and M. Miyayama, Appl. Phys. Lett. 78, 1903 (2001).

${ }^{4}$ Y. Goshima, Y. Noguchi, and M. Miyayama, Appl. Phys. Lett. 81, 2226 (2002).

${ }^{5}$ D. Mercurio, G. Trolliard, T. Hansen, and J. P. Mercurio, Int. J. Inorg. Mater. 2, 397 (2000).

${ }^{6}$ A. Snedden, D. O. Charkin, V. A. Dolgikh, and P. Lightfoot, J. Solid State Chem. 178, 180 (2005).

${ }^{7}$ Z. G. Yi, Y. X. Li, Z. Y. Wen, S. R. Wang, J. T. Zeng, and Q. R. Yin, Appl. Phys. Lett. 86, 192906 (2005).

${ }^{8}$ K. R. Kendall, J. K. Thomas, and H. C. zur Loye, Chem. Mater. 7, 50 (1995).

${ }^{9}$ J. T. S. Irvine, D. C. Sinclair, and A. R. West, Adv. Mater. (Weinheim, Ger.) 2, 132 (1990).

${ }^{10}$ C. Ang, Z. Yu, and L. E. Cross, Phys. Rev. B 62, 228 (2000).

${ }^{11}$ K. L. Ngai, Y.-N. Wang, and L. B. Magalas, J. Alloys Compd. 211/212, 327 (1994).

${ }^{12}$ X. P. Wang, Q. F. Fang, Z. S. Li, G. G. Zhang, and Z. G. Yi, Appl. Phys. Lett. 81, 3434 (2002).

${ }^{13}$ A. S. Nowick and B. S. Berry, Anelastic Relaxation in Crystalline Solids (Academic, New York, 1972), p. 265. 